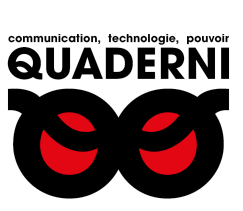

\title{
Quaderni
}

Communication, technologies, pouvoir

96 | Printemps 2018

Smart city : "fiction" et innovation stratégique

\section{Grenoble et la «Smart City ». Entretien avec Éric Piolle, maire de Grenoble}

\section{Kevin Brookes}

\section{(2) OpenEdition \\ 1 Journals}

\section{Édition électronique}

URL : https://journals.openedition.org/quaderni/1178

DOI : 10.4000/quaderni.1178

ISSN : 2105-2956

Éditeur

Les éditions de la Maison des sciences de l'Homme

\section{Édition imprimée}

Date de publication : 15 mai 2018

Pagination : 59-70

\section{Référence électronique}

Kevin Brookes, «Grenoble et la "Smart City ». Entretien avec Éric Piolle, maire de Grenoble », Quaderni [En ligne], 96 | Printemps 2018, mis en ligne le 15 mai 2020, consulté le 28 septembre 2021. URL : http://journals.openedition.org/quaderni/1178; DOI : https://doi.org/10.4000/quaderni.1178 


\section{$D$ ossier}

\section{Grenoble et la}

« Smart City » Entretien avec Éric Piolle, maire de Grenoble

Éric Piolle a été élu maire de Grenoble en 2014 à la tête d'une coalition rassemblant des écologistes et des membres du Parti de gauche et diverses associations citoyennes. Il a retenu l'attention du comité éditorial de Quaderni pour plusieurs raisons. Tout d'abord, car il est le seul élu écologiste à la tête d'une grande agglomération et ainsi en mesure de mettre en cuvre des mesures concrètes comme l'abaissement de la vitesse légale des voitures. Ensuite, car l'élection inattendue de sa liste hétéroclite (face à un candidat ex-adjoint PS de la ville) a créé les conditions d'une expérience politique inédite visant à favoriser la démocratie participative et l'implication de la société civile. Enfin, en raison de la distance critique qu'entretient Éric Piolle avec le concept de "Smart City», alors même que le territoire grenoblois est marqué par les secteurs de la recherche et des entreprises de nouvelles technologies.

Le terme «Smart City» est à la mode sur le plan médiatique. Que vous évoque-t-il?

La dimension du vocabulaire est importante. Pour moi, cela évoque quand même une sorte de mythologie technologiste de la ville, alors qu'en pratique d'ailleurs le contenu peut être beaucoup plus riche et créateur que cela. Quand on a créé les Smart Cities, les Smart Phones, on a eu un regard un peu décalé, où avec le vocabulaire on célèbre un peu le dieu technologie.

\section{Par Kevin Brookes}

Doctorant en science politique Laboratoire PACTE Science-Po Grenoble
Des institutions comme la Commission européenne essaient de développer le concept et présentent cela comme une solution possible pour répondre à la complexité et à l'urba- 
nisation croissante de nos sociétés. Si on l'emploie pour faire référence à des solutions concrètes de politiques publiques, la notion vous convainc-t-elle?

Derrière le vocabulaire qu'il faut détourner et questionner, effectivement le contenu peut être plus ou moins riche et peut être plus ou moins créateur d'espoir ou de solutions. Là-dessus, il y a finalement deux regards sans doute qui s'opposent, parfois se mélangent, et souvent se croisent sur la ville. D'un côté, un regard presque d'ingénieur, de planificateur qui cherche à répondre à une logique de flux. La ville devient complexe parce qu'il y a beaucoup de flux, parce que c'est un aspirateur à ressources - ressources alimentaires, en matières premières - c'est une usine à déchets absolument hallucinante avec des pointes extrêmes comme on peut avoir à New York où vous voyez ces chaînes de camion qui amènent des déchets à $1500 \mathrm{~km}$ de là. On a cette ville finalement dont on s'occupe pour gérer les flux. Et on a un autre regard sur la Cité comme espace de démocratie, espace de projet, espace de conflits, espace à la fois de projets d'individus et de collectifs qui ont en ville une échappatoire au contrôle social que pouvait représenter le village pendant toute une époque. Clairement, je ne pense pas que la ville soit une usine dont il faudrait optimiser les flux et les habitants ne sont pas des objets dont il faudrait aussi optimiser les flux, bien loin de là. Et pourtant, je mesure tout ce projet, parce qu'en plus ma carrière professionnelle est faite dans l'industrie et dans la supply chain : j'ai passé 18 ans à essayer d'optimiser des flux de matières, des flux d'informations, des flux financiers. Je retrouve parfois dans ce discours-là le même vocable, les mêmes logiques, et les mêmes réflexions que je pouvais avoir dans une sphère professionnelle avec des objets, de la matière, de la production de biens ou de services dans des usines, mais tout d'un coup on passerait à l'échelle d'une ville où les objets et les services seraient en fait des petits humains.

Justement, plusieurs traits semblent faire de Grenoble une Smart City. Par exemple, au niveau de la métropole, il y a un chef de projet Smart City, il y a des réalisations concrètes qui ont été faites comme l'installation de smart grids, la mise en ouvre de l'open data, ou l'ouverture de plateformes collaboratives. Comment sélectionnez-vous les dimensions de la Smart City qui vous semblent pertinentes et celles qui au contraire ne le sont pas?

Nous poussons tout ce qui va développer une émancipation du citoyen et sa capacité à prendre des décisions dans lesquelles il arbitre entre son intérêt personnel et l'intérêt collectif. C'est cela qui est intéressant. Et la technologie - on n'est absolument pas technophobe non plus - peut être mise au service de ce projet-là. Cela correspond pour moi aussi à une bascule sociétale plus forte : d'une société jacobine vers un monde d' «acteurs réseaux» dans lesquels on va retrouver du sens, notamment dans les échanges économiques. C'est-à-dire que l'évolution sociétale - pour reprendre les termes de Roger Sue - tend vers à la fois plus d'individus, mais aussi plus d'égalité réelle, sur une individuation des réflexions, tout en ayant un fort lien à l'altérité et donc la dimension collective. Cela se retrouve finalement dans une ville qui crée et avance dans cette société d'acteurs réseaux, en utilisant des moyens parfois technologiques, mais en gardant toujours 
une dimension en tête : il faut à la fois se saisir des opportunités technologiques (cela apporte des choses en termes de gestion de l'énergie, de mesure et d'informations), mais faire également qu'une ville reste low tech parce qu'elle doit être robuste.

\section{Low tech, c'est-à-dire?}

Low tech, c'est l'innovation frugale de Navi Radjou. On est dans cette logique de se dire que finalement la technologie doit aller très loin et on voit que maintenant la porosité entre la science et la technologie est souvent forte. D'un autre côté, il ne faut pas oublier qu'on est dans le monde qui est le nôtre, c'est-à-dire un monde qui fait face à des chocs qui vont être de plus en plus forts (on le voit avec les cyclones en ce moment). Une dimension très forte à la fois de démocratie et de vie humaine, c'est notre capacité collective à résister à ces chocs. Comment y résiste-t-on? Ce travail sur la résilience et la robustesse, il est important aussi, et l'innovation technologique low tech est forte. Navi Radjou a une problématique intéressante : il faut penser l'urbain également à l'échelle de la planète et les solutions qui marchent sont souvent des solutions suffisamment simples pour qu'elles se propagent vite et à des millions et à des millions de personnes.

Avez-vous une vision positive de certaines choses qui ont été faites auparavant à Grenoble notamment pour optimiser la consommation énergétique, par exemple l'installation de capteurs pour optimiser le suivi de ce type de flux? Est-ce que cela correspond à votre vision du low tech?
Oui, avec toujours un conflit que l'on porte assez fortement : il faut que cela soit accessible à tous. J'y veille en tant que président de bailleur social. Parce que parfois, ils font une sorte d'ordinateur de bord qui n'intéressera que les geeks et les bacs +12 , pour piloter la consommation énergétique d'un appartement. Or, moi ce qui m'intéresse, c'est que la vieille dame de 70 ans puisse se saisir de la question. Et on le voit dans les fournisseurs : certains ont développé des machins si intelligents, mais tellement intelligents qu'ils sont en fait non utilisables. Ou alors ils sont utilisables par des sociétés commerciales à l'extérieur qui vont venir vous vendre des services sur la base de vos informations. La question de la donnée privée et de la liberté des données est évidemment forte, tout comme la capacité des citoyens à prendre des décisions par eux-mêmes : il faut qu'ils puissent trancher leurs contradictions eux-mêmes. Pour moi, c'est fort en termes d'émancipation : il ne faut pas qu'ils transfèrent leurs contradictions aux autres. Exemple : on veut tous avoir de l'électricité pour pas cher en France, pomper sans réfléchir et puis en même temps, on a peur du nucléaire. Il faut que les citoyens tranchent leurs contradictions par eux-mêmes, plutôt qu'un monde surplombant.

Cela renvoie à un aspect de la Smart City : pour qu'elle fonctionne, il faut qu'il y ait des smart people pour utiliser des nouvelles technologies ou des dispositifs de politiques publiques de plus en plus complexes. Comment faire pour accompagner cette transition, pour finalement redonner du pouvoir aux citoyens face à la complexité de certaines technologies soutenues par les municipalités? 
Alors nous, je ne pense pas qu'on soutienne des innovations pour lesquelles il faut qu'on ait des smart people. Ou alors, c'est dans une vocation de recherche, c'est-à-dire qu'on joue à cela typiquement dans certains domaines où l'objectif est celui de recherche d'opérationnalité : il faut le tester et quand on teste quelque chose, on peut se permettre qu'il soit testé auprès des personnes qui sont plus sensibles au sujet pour qu'ils nous fassent des retours. Mais à la fin, il faudra que cela se concrétise quand on changera d'échelle : il faut que cela soit suffisamment low tech pour être accessible à tous. La ville est aussi un espace d'expérimentation.

\section{Comment faire en sorte qu'avec l'installation de nouveaux compteurs, de nouvelles appli- cations, de nouvelles technologies démocra- tiques, que le fossé ne se creuse pas entre les plus richement dotés en capital culturel et social et ceux qui le sont moins?}

En effet, comment faire en sorte que l'on n'ait pas d'un côté des outils smart cities pour smart people et de l'autre côté que l'on ait de plus en plus d'écrivains publics qui sont là pour être des médiateurs entre des habitants et leurs droits ou services? Parfois, il y a la barrière de la langue, parfois la barrière technologique. Cette loi du plus fort, on est tenté de dire que cela va marcher. Les «winners» d'aujourd'hui qui sont dans le «move» et qui se sentent à l'aise avec toutes ces technologies disent finalement : "c'est ça qu'il faut pour tout le monde». Dans cette logique qui s'apparente à la loi du plus fort, ils oublient qu'ils seront les «paumés» de demain. Ils ont beau être au top de la geekerie aujourd'hui et bien dans 30 ans, il y aura des nouvelles technologies, des nouveaux outils et ils regarderont cela comme une poule regarde un couteau. Je pense qu'on voit toutes les solutions que la technologie peut apporter et c'est donc évidemment intéressant. La question importante est de voir comment cette technologie peut soutenir cette génération d'acteurs réseaux et ainsi favoriser l'émancipation et donner du sens dans l'échange. Quelques exemples : j'échange l'énergie avec le quartier voisin, car ça y est j'ai autoconsommé la mienne et j'ai un excédent ou un besoin; j'échange l'alimentation parce que j'ai développé ce que je pouvais en termes de souveraineté alimentaire, mais j'aime bien manger des bananes et je vais échanger des bananes avec les pommes que je fabrique ici et qu'ils n'ont pas là-bas. Du coup, il y a du sens dans l'échange. Il faut toujours faire attention à cela. $\mathrm{Si}$ on a un regard uniquement technophile, on oublie les grands enjeux et on se dit que les chercheurs vont nous trouver des solutions au dérèglement climatique. Donc, on repousse à plus tard et on va dans le mur. L'histoire a montré que l'usage de la technologie et de l'industrie pouvait parfois se retourner. Le rôle de l'industrialisation dans les deux guerres mondiales du $\mathrm{XX}^{\mathrm{e}}$ siècle et le développement chimique de l'agriculture d'après-guerre n'est pas anodin. On sait qu'il y a un moment où cela peut basculer vers quelque chose qui n'est totalement pas démocratique et qui peut même nous entraîner vers la guerre. Et il faut garder cela évidemment en tête.

Justement, je voudrais revenir avec vous sur l'idée d' «acteurs réseaux» en décomposant la notion de Smart City. Sur la partie gouvernance, votre projet serait d'éviter d'avoir une vision verticale imposée par la munici- 
palité, et de développer des «acteurs réseaux». Comment utiliser les nouvelles technologies pour favoriser cette mise en réseau? Qu'avez-vous fait depuis votre élection pour la favoriser?

Cela peut être à travers la technologie ou à travers le cadre concret. Comment regardons-nous notre rôle? En tant que leader politique, je ne suis pas en train d'imposer un imaginaire qui serait le mien. J'ai une fonction où je dois poser le cadre qui permet de s'assurer que l'on va combiner nos imaginaires individuels. L'important c'est de voir comment on les conjugue pour produire un imaginaire collectif qui nous permette d'avancer. Il s'agit d'une grande fonction avec derrière des fonctions d'exécution. C'est-à-dire des fonctions de cadrage et d'arbitrage avec des processus d'apprentissage permanent et d'apprentissage collectif. Il y a aussi une fonction de rôle modèle, d'exemplarité, en sachant que l'on a tous nos incohérences et que personne n'est exemplaire. Il faut que les gens puissent identifier les discours que je porte avec la manière dont ils me voient vivre et qu'il n'y ait pas de déconnexion majeure de manière à ce que mon discours soit balayé. C'est cela finalement mes quatre fonctions de maire. On pourrait dire que c'est ce que l'on fait par exemple sur la démocratie locale en développant des votations citoyennes sur des budgets participatifs. On le fait par différents biais : on essaie d'avoir des forums de contributeurs dans tous les exercices de concertation : on passe à la fois par internet, mais aussi par le physique et par le papier. En fait, il faut multiplier les hameçons, les pistes parce qu'on n'a pas tous le même rapport à la participation. Quand on faisait des présentations de projets, on savait bien que pour certains il faut des graphes parce que c'est cela qui va leur parler, pour d'autres, il faut du texte à lire, pour d'autres il faut de l'oral. Cela ne dit rien des qualités, cela dit juste qu'on est différents et qu'on n'a pas les mêmes médiums d'apprentissage et d'échange. Finalement la technologie est un moyen comme un autre de mettre en rapport les citoyens.

Souvent, dans les municipalités à la pointe des technologies de la smart city, il y a utilisation des smartphones comme outils citoyens. Par exemple, pour signaler des problèmes d'entretien. Est-ce le type de technologies qui vous parlent?

Oui, je trouve cela intéressant. On a un projet qui repense la relation citoyenne et qui est un moyen d'adapter le service public aux besoins et aux envies des gens. Beaucoup de personnes qui travaillent n'ont pas le temps de passer aux réunions publiques et sont à l'aise avec les smartphones et sont contentes de pouvoir contribuer en envoyant une photo de quelque chose qui ne va pas en ville. Cela va nous permettre de traiter de manière plus rapide toute cette partie-là et de pouvoir aussi assigner des moyens à ceux qui ont envie de venir discuter 5 minutes, se faire expliquer quelque chose, ou ceux qui souhaitent pouvoir dire directement à une personne que la plaque d'égout est mal scellée dans leur rue. Pour moi, cela permet d'assigner mieux nos ressources.

\section{Pouvez-vous expliquer en quoi consiste votre projet «Grenoble ville de demain »?}

L'objectif, c'est la porosité. Une des caractéris- 
tiques de Grenoble est sa capacité de mélange et d'intégration de diasporas successives qui sont venues ici, qu'elles soient savoyardes, espagnoles, chiliennes, arméniennes, et puis maghrébines, etc. et qui ont fait la ville depuis toujours. Et cette capacité de mélange, elle se retrouve dans « Ville de demain » où on veut s'appuyer à la fois sur les étudiants, le monde universitaire, les associations, les collectifs, le monde économique pour penser les sujets de façon transversale. Il y a des moments qui s'appellent «la fabrique» où il y a souvent des étudiants avec quelques profs qui ont produit une première matière qui va être discutée. Il y a eu par exemple une fabrique sur les îlots de chaleur en ville, une autre sur le mobilier urbain. Ce sont à la fois des projets de réflexion, mais aussi d'actions concrètes sur comment on fait la ville. Il y a aussi cette vocation d'accueillir tous les gens qui veulent venir travailler avec nous de l'extérieur. En effet, il y a beaucoup de gens qui ont envie de travailler avec nous, en France et partout dans le monde parce qu'il se passe ici des choses un peu différentes. Ils se disent qu'il y a là un champ d'expérimentation. Cela permet de contribuer à mélanger ces gens et faire en sorte qu'ils en retirent quelque chose de valorisant à la fois pour leurs travaux et pour la ville en traduisant cela dans des projets concrets sur le terrain. Qu'est-ce qu'on fait pour la qualité de l'air? Cela se traduit concrètement par des mesures politiques. Qu'est-ce que l'on fait sur l'information? Cela va se traduire dans ce que l'on fait pour l'information culturelle aujourd'hui en ville. Qu'est-ce qu'on fait pour l'économie sociale et solidaire? Enfin, il y a pas mal de choses qui se traduisent derrière dans l'action. On arrive à dépasser un peu qui on est et notre historique pour rencontrer l'autre et faire quelque chose.
Ce sont des réunions et l'outil internet et numérique n'est que secondaire, ce n'est qu'un moyen?

Ce n'est qu'un moyen. De même que l'agriculture urbaine est à la fois un objectif et un moyen. C'est un objectif pour moi de robustesse et de résilience de la ville. Mais c'est un moyen de retrouver un sens à notre alimentation et donc de retrouver un lien aussi avec la nature, même en ville. Et c'est un moyen que des gens très différents se rencontrent, parce que quand ils jardinent ils ne se préoccupent pas de savoir qui est ingénieur, qui est retraité de la chimie, qui est au chômage, ils font quelque chose ensemble et ils passent un bon moment ensemble avec un objectif commun.

Justement, on va aborder avec vous cette question des fins que vous recherchez en tant qu'écologiste et comment justement cela peut être en opposition avec la Smart City. J'aimerais revenir notamment sur la question du développement durable. Vous avez déjà récusé ce que vous qualifiez de «vision techniciste des choses» qui consisterait à gérer la consommation énergétique de la ville en gestion de flux. Quelle vision alternative proposez-vous pour résoudre les problèmes environnementaux et de pollution qui se posent particulièrement à Grenoble?

Nous sommes passés à $100 \%$ d'énergie verte depuis le $1^{\text {er }}$ janvier 2016 sur les équipements communaux. Nous avons mis en place notre plan de protection de l'air comprenant des aides pour la conversion de système de chauffage en bois afin d'aller vers des systèmes sans ou avec une forte réduction des émissions de particules. 
Au niveau de la circulation, nous avons mis en place une restriction à la circulation automobile et une incitation aux transports en commun ou aux modes doux - ou aux modes «actifs» comme on les appelle. Il faut aussi mettre en place des projets de recherche. On a un projet avec Bouygues là - parfois on a des alliés qui ne sont pas forcément nos alliés naturels - qui travaillent sur l'autoconsommation collective. J'étais à la C.R.E $E^{1}$ la semaine dernière pour pousser à des expérimentations sur l'autoconsommation collective, parce que c'est cela qui permet de développer des projets d'énergie renouvelables. Il s'agirait de mettre en place un pilotage des biens communs par le citoyen. La dimension municipale a cela d'intéressant qu'elle part des biens communs concrets : l'eau, l'énergie, les déchets, l'assainissement. Or, on peut mettre ces biens communs sous un pilotage de citoyens, on aurait là une instance éminemment démocratique. Si on met en place un comité des usagers de l'eau, un comité des usagers du chauffage urbain, il va y avoir des citoyens qui vont s'investir, se passionner pour le sujet, et développer des compétences parfois plus grandes que des techniciens ou avec des regards différents. Cela relève là typiquement d'une appropriation du bien commun par les citoyens et c'est donc éminemment démocratique. C'est un objectif en tant que tel : la démocratie, donc la liberté. Et c'est un moyen, en ce que cela permet de changer d'échelle. Les changements ne viennent pas d'en haut, parce qu'en haut on a été formé en masse sans doute dans les mêmes sphères, il y a longtemps, il y a une contrainte de lobbys, il y a beaucoup de freins au changement, on prend les sujets les uns après les autres, donc cela avance très lentement. Alors que quand on se dit que cela va partir d'en bas et bien cela foisonne. D'un côté, on ne contrôle pas - mais, c'est cela qui est intéressant, c'est toute la dimension de liberté - et de l'autre côté cela avance beaucoup plus vite, parce que les citoyens vont beaucoup plus vite que les institutions.

Pour vous, développer la démocratie à tous les niveaux permet au niveau le plus local de faire avancer les choses grâce à l'implication des citoyens. Mais vous avez aussi évoqué un autre acteur : Bouygues. Les Smart Cities, en théorie, c'est aussi le renforcement des partenariats publics - privés, et là vous avez cité un exemple concret. Comment faites-vous pour faire en sorte qu'il y ait un équilibre entre les acteurs privés, les élus, mais aussi les citoyens?

Comme tous les équilibres, c'est fin et c'est une expérience de terrain. Pour donner un exemple, il y a beaucoup de sociétés qui ont créé des départements de développement durable. Souvent au départ par "Green Washing», parce que c'est à la mode, ou parce qu'un boss a été sensibilisé à cela. Mais derrière, ces organisations ont été transformées : elles ont assigné des ressources et de l'intelligence à cela. Typiquement, travailler aujourd'hui avec des entreprises comme celles-là permet aussi de les transformer de l'intérieur. Je vois les pratiques de Bouygues sur le terrain - cela n'empêche pas ce qu'ils font par ailleurs - et il n'empêche qu'ils assignent ces ressources-là et donc cela transforme leur regard sur les choses.

\section{Vous parliez d'«ingérence du privé» dans votre programme électoral...}

Tout à fait, mais sur le contrôle des biens communs... 


\section{Mais comment l'éviter?}

On a écarté de la direction générale de notre compagnie de chauffage, donc de notre réseau de chaleur, le directeur général qui venait de l'actionnaire minoritaire privé. On a fait pareil pour notre distributeur local d'électricité et de gaz GEG. Voilà le pilotage qui est fait sur la gestion des biens communs. Ce qui n'empêche pas la coopération avec le privé. Sur la production d'énergies renouvelables qui est extrêmement capitalistique et dans lequel il y a des évolutions technologiques fortes, on travaille avec des entreprises privées. Cela ne me gêne pas du tout. Elles amènent quelque chose. Il faut juste mesurer ce qu'elles apportent et être conscient du fait qu'en tant qu'organisations elles ont des intérêts - sans remettre en cause l'intégrité et la sincérité des gens que l'on a en face de nous. Ces intérêts s'incarnent dans un triptyque : freiner, édulcorer et récupérer. Elles freinent les avancées qui peuvent les gêner. Une fois qu'elles ne peuvent plus les freiner, elles les édulcorent, elles cherchent à les saboter. On l'a vu après le Grenelle de l'environnement. On l'a vu avec les panneaux publicitaires : il y avait beaucoup de choses intéressantes qui avaient été produites. Puis, cela a été édulcoré et les entreprises ont finalement siphonné le sujet, et puis derrière, une fois qu'elles ne pouvaient plus faire autrement, elles ont récupéré et rebâtit la barricade 50 mètres en arrière et puis le jeu est reparti. Mais c'est la vie. On a un regard lucide. Ce n'est pas un blancseing, mais on a des espaces d'intérêt commun sur lesquels il faut avancer.

Je souhaiterais revenir avec vous sur le projet City Zen. L'idée générale est de faire du mo- nitoring territorial sur l'optimisation énergétique. Est-ce que vous pourriez dire quelques mots sur ce genre de projets qui consistent à développer des données pour que les acteurs gèrent leur consommation énergétique?

Évidemment dans le domaine de l'énergie, on a une question qui est la consommation, une question qui est la production, enfin la question d'allier production et stockage. Ce sont les grands enjeux que l'on retrouve dans les programmes qui nous influencent fortement, comme «Megawatt» sur les questions de sobriété énergétique, d'efficacité énergétique et d'énergies renouvelables. C'est le triptyque que l'on met en avant. Dans cette logique, le pilotage des consommations, l'information et la diffusion d'informations permettent généralement la sobriété. Si je vois à quel point mon frigidaire consomme, peutêtre vais-je le changer, le nettoyer pour qu'il consomme moins ou me dire que je n'ai pas besoin d'avoir un réfrigérateur de 500 litres. On a vu les effets rebonds : on consomme moins, mais nos objets surconsomment quand même, car ils changent de taille et changent d'échelle. On se donne aussi les moyens de dire comment, à une échelle territoriale, on peut déjà assurer des boucles locales qui soient efficaces. Quand on a eu le risque de délestage électrique en janvier dernier, on a informé la population, on a informé les endroits où on allait couper. Cela développe aussi de la résilience parce qu'on ne va peut-être pas tous lancer le lave-vaisselle à $20 \mathrm{~h}$ après dîner ou notre lave-linge à $18 \mathrm{~h}$ quand on rentre. Cela permet de donner des moyens aux habitants de choisir leurs comportements et d'être conscients des conséquences de leurs comportements. Cela accentue cette liberté-là. Et donc derrière on a 
aussi cette réflexion sur cette question de l'autoconsommation et de la boucle locale.

\section{Les informations en question sont des don- nées produites, centralisées et gérées par des entreprises?}

Cela dépend. On en est à une phase d'expérimentation du projet où l'intérêt est de capter la donnée pour comprendre ce qui se passe. Si on veut avancer, il faut quand même investir aussi dans une dimension d'apprentissage et de compréhension de ce qui se passe. Après, il faut juste s'assurer que ces données, une fois que l'on sort de l'expérimentation, ne seront pas utilisées et la protection des données privée sera forte. C'est-à-dire que je ne peux pas en profiter pour mesurer quand vous êtes chez vous et vous envoyer de la publicité adaptée quand vous êtes en train d'allumer votre four - parce que l'on peut mesurer cela. Et il faut faire en sorte que personne ne soit en train de vous vendre l'information que l'on vous a prise. L'enjeu fort, c'est ça. Mais par contre dans les phases en amont où l'on s'interroge sur la manière dont fonctionne un quartier et comment on collecte ces données sont intéressantes.

\section{La solution serait donc pour vous de mettre en place l'autoconsommation collective?}

C'est une piste d'expérimentation intéressante bien sûr parce que l'on peut baisser la consommation énergétique. Vous savez dans l'énergie, comme dans la supply chain en général, il y a la question de la consommation moyenne et des pics. Si on veut faire de la sobriété, il faut redéfinir nos moyens, mais il faut aussi adapter nos comportements pour éviter les pics. Ces derniers génèrent des surconsommations et ne sont pas bons pour le système. Si à l'échelle d'un immeuble - tel qu'on va le faire avec l'immeuble «ABC»-on peut avoir une buanderie commune et puis s'assurer que l'on ne lance pas tous notre lave-linge au même moment, et bien on aura beaucoup gagné. De même que dans le domaine des transports, les réflexions qui sont menées pour lisser les horaires des démarrages des entreprises peuvent changer les choses. Je faisais pareil dans mon travail quand j'expédiais des colis pour le service après-vente dans le monde entier : je faisais en sorte d'éviter que tout le monde appelle à 4 heures et demie pour avoir son colis expédié à $18 \mathrm{~h}$. En travaillant sur la demande, on lisse les choses. C'est le bullwhip effect, c'est-à-dire l'effet lasso. C'est une théorie intéressante, car assez parlante, à la base sur la consommation des couches-culottes. Par définition, vous avez un nombre d'enfants en bas âge, quelques personnes âgées, mais le signal de sortie, le besoin est extrêmement stable, il est linéaire. En pratique, des spécialistes avaient modélisé toute la supply chain des couches-culottes. À partir d'un besoin stable, les pratiques de passation de commande, d'abord de ceux qui achetaient les couches, les parents (le consommateur) puis du revendeur, puis du grossiste, puis du distributeur, puis du fabricant, faisaient qu'il y avait une saisonnalité monstrueuse sur la production des couchesculottes. C'est pour cela que l'on appelle cela le bullwhip effect : c'est le lasso, car à partir d'un signal qui est donc la pointe du lasso qui est plat, et bien on se retrouvait avec une onde extrêmement forte à l'autre bout, et une saisonnalité. Et donc il faut travailler sur l'information, en redéfinissant d'abord quel est le besoin réel : comment faut-il 
que je lave mon linge, ma vaisselle (si possible dans un lave-vaisselle)? Quels sont mes besoins électriques, de chauffage, de douches et sanitaires et de mobilité? En repensant nos besoins de base et en diffusant cette information partout sur la chaîne, finalement je vais m'apercevoir que je peux lisser cette demande et éviter d'avoir un signal de consommation, de nécessité de production électrique trop fort à l'entrée.

\section{Si j'ai bien compris, l'idée serait de permettre aux citoyens de lisser leur consommation au niveau le plus local possible?}

Oui et en tout cas c'est une expérience intéressante et on voit comment on s'entraîne à faire ces choses-là. C'est là-dedans que l'on peut développer pour moi de la liberté. Les citoyens cultivent leur liberté en faisant en sorte qu'ils soient en mesure d'arbitrer entre la conscience qu'ils ont de leurs besoins réels et l'intérêt général. C'est un regard qui est plutôt optimiste sur la nature de l'homme. En effet, si je me dis que l'homme est un crétin qui ne pense qu'à lui et qui se préoccupe peu de la planète, je me dis qu'on va changer de système. C'est le modèle des smart cities technicistes où l'on pilote cela à la place des citoyens parce qu'ils ne seraient pas en mesure de le faire eux-mêmes.

Dans quelle mesure pensez-vous que ce type de technologies puissent être mises au service de la réduction des inégalités sociales? Comment intégrer votre vision en faisant en sorte que dans des villes qui sont très technologiques, où il y a une part très importante de diplômés, on évite justement ces inégalités en capital culturel?
Pour donner un exemple, nous sommes en train de travailler sur le projet de l'ANRU2 en nous assurant que les habitants des quartiers prioritaires du ministère de la Politique de la Ville aient les moyens de se saisir des enjeux du $\mathrm{XXI}^{\mathrm{e}}$ siècle sur la question foncière, de matière première, d'énergie, de biodiversité, etc. Ils sont autant contributeurs que les autres. Pour moi, c'est l'inverse de la politique du ruissellement qui est mise en œuvre par le gouvernement où l'on dit «libérons l'énergie des riches et des innovants, cela finira par couler sur les boulets qui sont en bas». Avec nous, c'est tout l'inverse. Dans mon quotidien de maire, je rencontre dans la même journée des gens qui ont peu de capital culturel en lien avec le système et à l'autre bout du spectre des gens qui sont au top dans ce domaine-là. Je suis émerveillé en permanence de leur humanité commune, des mêmes questions qu'ils se posent ensemble. À la fin, vous avez beau être le patron de Facebook, être au fond d'une situation de précarité économique, eh bien vous vous posez les mêmes questions. Votre humanité c'est la même. C'est mon expérience de maire au quotidien. La technologie, elle, est asociale. Si elle est prise par des puissants, elle sera au service des puissants, si elle est saisie par des enjeux de justice sociale, elle permettra d'avancer dans ce sens. Pourquoi pas. Pour moi, les technologies ne sont pas que des outils, ce qui compte c'est comment on les utilise et comment on s'entraîne avec. C'est un entraînement. Notre capacité de liberté est un entraînement permanent. Je peux cultiver ma liberté ou cultiver mon asservissement au modèle économique dominant. C'est mon choix de tous les jours. Ce sont aux citoyens de décider. La liberté ne se donne pas, elle s'arrache. 


\section{$N \cdot O \cdot T \cdot E \cdot S R \cdot E \cdot S \cdot U \cdot M \cdot E$}

1. [NDLE] Autorité administrative indépendante chargée de veiller au bon fonctionnement des marchés de l'électricité et du gaz en France.
Éric Piolle a été élu maire de Grenoble en 2014 à la tête d'une coalition rassemblant des écologistes, des membres de « La France Insoumise » et de diverses associations citoyennes. Dans cet entretien, réalisé en septembre 2017, il nous expose ses critiques vis-à-vis d'une conception trop technophile et élitiste de la «Smart City». Ce vocable polysémique est souvent utilisé pour faire du maire un gestionnaire central d'un certain nombre de flux (énergétiques, économiques, etc.). Sans rejeter l'intérêt que présentent certaines innovations technologiques pour l'administration des communes, il oppose à cette démarche verticale une démarche alternative. Les technologies doivent être mises au service d'un projet émancipateur pour des citoyens informés et capables d'arbitrer entre leur intérêt individuel et l'intérêt général. Ceci passe notamment par leur implication plus grande dans le débat public, mais également par leur participation à des instances de gestion énergétique. Cette vision n'est pas empreinte de certaines ambiguïtés, particulièrement quant à la place des entreprises privées. Elles sont considérées par Eric Piolle comme des alliées, mais avec lesquelles il faudrait une relation critique car elles incarnent des intérêts particuliers parfois en tension avec les projets de la ville.

\footnotetext{
Abstract

Éric Piolle was elected mayor of Grenoble in 2014 as a leader of a coalition of ecologists, members of " $\mathrm{La}$ France Insoumise" (left), and various associations of citizens. In this interview conducted in September 2017, he presents his criticisms of an elitist and technophile vision of the "Smart City." This fuzzy concept is often used to consider the mayor as a central manager of a certain number of flows (energetical, economical
} 
etc...). However, Eric Piolle does not fully reject the benefits of some technological innovations for the good management of cities. Against the top-down approach of the "Smart City" he offers an alternative and bottomup approach. For him, technologies must be put to work for the benefit of an emancipating project for citizens who would become more informed to rule between their personal and the public interest. This implies that they get more involved in the public debate, but also in local bodies of energy management. This vision is not free from several ambiguities, especially as far as the place of private firms are concerned. They are considered as allies, but it implies a critical and distant relationship at the same time because of the tension between their vested interest and the projects of the city. 